\title{
Women in Academic Psychiatry: Inequities, Barriers, and Promising Solutions
}

\author{
Marcy Forgey Borlik ${ }^{1}$ (I) $\cdot$ Sarah M Godoy ${ }^{1} \cdot$ Paula M Wadell $^{2} \cdot$ Lidija Petrovic-Dovat $^{3} \cdot$ Consuelo C Cagande $^{4}$. \\ Aditi Hajirnis $^{5}$. Eraka P Bath ${ }^{1}$
}

Received: 1 June 2020 / Accepted: 22 December 2020 / Published online: 2 February 2021

(C) Academic Psychiatry 2021

For the first time in US history, first-year female medical school matriculants $(50.7 \%)$ outnumbered men $(49.3 \%)$ in 2017 [1]. Moreover, in 2019, women accounted for 50.5\% of all medical students for the first time [1]. Yet, female faculty continue to be underrepresented at the highest rankings in academic medicine as a whole and in psychiatry $[2,3]$. Women represent only $26 \%$ and $32 \%$ of full professors among all medical faculty and psychiatry faculty, respectively, with a majority identified as White [3]. Structural racism, gender bias, and discrimination, along with the lack of systematic strategies that aim to achieve gender and racial equity, result in persistent achievement and promotion disparities among students, residents, and faculty, especially among those who are underrepresented in medicine $[4,5]$.

We will review the barriers women face advancing their careers in academic medicine in general, and academic psychiatry in particular, with specific attention paid to inequities for Black, Indigenous, and People of Color (BIPOC) women and especially underrepresented in medicine (URM) women compared to White women based on race/ethnicity. We will also consider the intersecting impact of sexual orientation and gender identities on women. Although there is a substantial body of research on academic medical career progression for

Marcy Forgey Borlik

mforgey@mednet.ucla.edu

1 David Geffen School of Medicine at The University of California, Los Angeles, Los Angeles, CA, USA

2 The University of California Davis School of Medicine, Davis, California, USA

3 Penn State College of Medicine, Hershey, PA, USA

4 Perelman School of Medicine, University of Pennsylvania, Philadelphia, PA, USA

5 Warren Alpert Medical School, Brown University, Providence, RI, USA women and URM, research identifying strategies and challenges for URM women is limited. Challenges noted include institutional barriers related to mentoring, time management, influence of bias, exclusion from formal and informal networks, and involvement in committees and non-promotion activities. Notably, the literature often considers women homogenously and does not account for nuanced differences between groups. Still, we propose solutions to narrow persistent gender and racial/ethnic disparity gaps for womenidentifying faculty.

The Association of American Medical Colleges defines underrepresented in medicine (URM) as "those racial and ethnic populations that are underrepresented in the medical profession relative to their numbers in the general population" [6].We use the term "URM women" to describe these women, who include all who do not identify as exclusively White or Asian [6]. We use the term "Black, Indigenous, and People of Color (BIPOC) women" to describe women whose racial/ ethnic identities are non-White to recognize the significant past and present history of violence, structural racism, and injustice toward Black and Indigenous people in the USA. We use the term "LGBTQIA+ women" to refer to lesbian, bisexual, transgender, queer, intersex, asexual, and all sexual and gender minoritized women in recognition of the discrimination and oppression they experience.

\section{Inequities and Barriers to the Advancement of Women}

While women comprised $42 \%$ of all US medical school faculty in 2019 , BIPOC women represented only $15 \%$ of all faculty, and URM women represented only $6 \%$ of all faculty [3]. The percentage of BIPOC women among women faculty by race/ethnicity was as follows: $20 \%$ Asian, $5 \%$ Black, $3.4 \%$ Hispanic, $2.5 \%$ multiple race Hispanic, $2 \%$ multiple race nonHispanic, and less than $1 \%$ identified as American Indian/ 
Alaskan Native or Native Hawaiian/other Pacific Islander [3]. From 2010 to 2019, the representation of Asian women among all female faculty has only increased by $3 \%$, while the percentage of Black female faculty did not increase [3]. Although there are more BIPOC women in medicine than ever before, they are commonly in lower ranks and less likely to have tenure or leadership positions than White women [3]. Further, URM women continue to be severely underrepresented as medical school applicants, matriculants, and at all academic ranks with progress that is slow or regressive and not proportionate to their respective populations $[3,7]$.

While all women are underrepresented in medical leadership, BIPOC, especially URM, women are more underrepresented than White women. In 2019, women represented only $19 \%$ of all US medical school chairs; yet, BIPOC and URM women represented only $24 \%$ and $15 \%$ of female chairs and $5 \%$ and $3 \%$ of all chairs, respectively [3]. A 2016 review of 135 medical institutions underscored the lack of women in decanal leadership, representing only $15 \%$ of all deans. Further, women deans were less likely to occupy roles related to general, research, clinical, and corporate decision making; disproportionately fulfilled educational, mentorship, and institutional public image needs; and experienced decreased representation with ascending decanal status [2]. Further, women represented only $18 \%$ of all US medical school deans in 2019, with only a $6 \%$ increase in representation as deans over 10 years [3]. Data for decanal race/ethnicity was not readily available.

All women academic physicians face discriminatory practices, such as lower compensation, slower promotion rates, and inconsistent or non-comprehensive paid family leave policies [7-9]. Women medical researchers are less likely than men to receive independent funding or publish in high-impact journals $[10,11]$. Women are less likely to be introduced by professional titles and are not well represented among prestigious awards or journal board and medical society leadership roles [11, 12]. Inequities are even greater when taking into account race, ethnicity, gender identity, and sexual orientation and are especially pronounced for BIPOC and LGBTQIA+ women, who must deal with significant structural inequality, bias, and oppression as they attempt to advance their careers.

\section{Women's Representation in Academic Psychiatry}

While representation of women in academic psychiatry has increased among junior faculty, their representation declines at higher ranks, with women accounting for only $34 \%$ of full professors in 2019, the majority of whom are White [3]. Table 1 shows the number and relative percentages of women among psychiatry faculty by race/ethnicity at each rank [3]. In psychiatry, URM women continue to be significantly underrepresented at every academic rank [3]. Further, while $23 \%$ of 2019 psychiatry chairs were women, BIPOC women represented only $6 \%$ of psychiatry chairs and URM women only $4 \%$ [3].

BIPOC women are more underrepresented than White women in terms of tenure. Among psychiatry faculty in 2019, there were 587 White men tenured compared to 281 White women, and only 19 Asian females tenured compared to 56 Asian men [3]. Black and Hispanic women had more representation in tenured positions than their male counterparts who were also underrepresented; specifically, in 2019 there were 13 Black female faculty tenured compared to 12 Black males, and 16 Hispanic female faculty tenured compared to 13 Hispanic males [3]. Still, a large proportion of Asian, Black, and Hispanic women were not on track for tenure at academic institutions that offer tenure track positions (543, 227, and 174, respectively) compared to their male counterparts $(500,110$, and 135 , respectively); AAMC data does not clarify or explain these differences [4]. Clearly, BIPOC women are not keeping pace with the career gains of White women [3, 24].

\section{Gender Salary Inequities}

Significant gender salary inequities exist across the academic medicine career span. Still, empirical data is limited, especially when accounting for women's race, ethnicity, and sexual orientation. In a review of department chairs $(N=1073)$ within 29 public medical schools, only 92 chairs (16.7\%) were women, including six female and 25 male psychiatry chairs [14]. Among all chairs, the unadjusted average difference in annual salary by sex was $\$ 79,061$. After adjusting for term length, specialty, inflation, title, and cost of living, the salary difference was $\$ 67,517$ [14]. The salary difference by sex for chairs serving for more than 10 years was $\$ 127,411$ [14]. AAMC 2018 faculty salary survey data showed mean male to female compensation differences of $20 \%(\$ 138,500)$ for all clinical science chairs, and $11 \%(\$ 53,400)$ for psychiatry chairs in US medical schools [15]. AAMC noted that although the faculty salary survey data may highlight national trends, critical data to determine equity such as time in rank and productivity are missing [15] (Table 2).

The 2018 AAMC salary survey results for clinical science physicians also showed sex differences in mean compensation, with men receiving higher compensation than women at all ranks [15]. The compensation differences (gaps) between male and female physicians in academia were as follows: among all clinical faculty (clinical instructor, $16 \%$; assistant professor, $23 \%$; associate professor, $24 \%$; professor, $21 \%$ ) and among psychiatry faculty (clinical instructor, $10 \%$; assistant professor, $8 \%$; associate professor, $9 \%$; professor, $8 \%$ ) [15]. The overall gaps were wider than within specialty gaps for all specialties, possibly influenced by gender specialty choice [15]. Since 2013, some gaps have narrowed, while others have widened [15]. (Table 2) Faculty survey data 
Table 1 Women faculty in psychiatry by rank/ethnicity

\begin{tabular}{|c|c|c|c|c|c|c|c|c|c|c|c|c|}
\hline \multirow{2}{*}{$\begin{array}{l}\text { Race/ethnicity } \\
\text { American Indian or Alaskan Native }\end{array}$} & \multicolumn{2}{|c|}{ Professor } & \multicolumn{2}{|c|}{ Associate professor } & \multicolumn{2}{|c|}{ Assistant professor } & \multicolumn{2}{|c|}{ Clinical instructor } & \multicolumn{2}{|c|}{ Other } & \multicolumn{2}{|l|}{ Total } \\
\hline & 0 & $0 \%$ & 0 & $0 \%$ & 6 & $0 \%$ & 3 & $0 \%$ & 0 & $0 \%$ & 9 & $0 \%$ \\
\hline Asian & 39 & $2 \%$ & 86 & $4 \%$ & 462 & $8 \%$ & 73 & $5 \%$ & 17 & $7 \%$ & 677 & $6 \%$ \\
\hline Black or African American & 15 & $1 \%$ & 43 & $2 \%$ & 183 & $3 \%$ & 41 & $3 \%$ & 3 & $1 \%$ & 285 & $3 \%$ \\
\hline Hispanic, Latino, or of Spanish origin & 20 & $1 \%$ & 28 & $1 \%$ & 139 & $2 \%$ & 48 & $4 \%$ & 2 & $1 \%$ & 237 & $2 \%$ \\
\hline Native Hawaiian or Pacific Islander & 0 & $0 \%$ & 0 & $0 \%$ & 3 & $0 \%$ & 2 & $0 \%$ & 0 & $0 \%$ & 5 & $0 \%$ \\
\hline Multiple race: Hispanic & 8 & $0 \%$ & 19 & $1 \%$ & 87 & $2 \%$ & 15 & $1 \%$ & 2 & $1 \%$ & 131 & $1 \%$ \\
\hline Multiple race: Non-Hispanic & 17 & $1 \%$ & 19 & $1 \%$ & 62 & $1 \%$ & 15 & $1 \%$ & 0 & $0 \%$ & 113 & $1 \%$ \\
\hline Other & 0 & $0 \%$ & 7 & $0 \%$ & 30 & $1 \%$ & 15 & $1 \%$ & 0 & $0 \%$ & 52 & $0 \%$ \\
\hline Unknown & 14 & $1 \%$ & 46 & $2 \%$ & 318 & $6 \%$ & 81 & $6 \%$ & 18 & $8 \%$ & 477 & $4 \%$ \\
\hline White & 547 & $28 \%$ & 694 & $36 \%$ & 2081 & $36 \%$ & 604 & $45 \%$ & 98 & $41 \%$ & 4024 & $36 \%$ \\
\hline Total women faculty & 660 & $34 \%$ & 942 & $49 \%$ & 3371 & $59 \%$ & 897 & $66 \%$ & 140 & $59 \%$ & 6010 & $54 \%$ \\
\hline Total all faculty & 1965 & & 1920 & & 5708 & & 1352 & & 238 & & 11183 & \\
\hline
\end{tabular}

Source: AAMC Faculty Roster, 2019

Percentages are calculated out of total of all faculty per rank

combining sex with race/ethnicity was not readily available. Further, women researchers are impacted by gender inequities in funding, career development awards, and start up packages $[8,16]$.

Available research suggests that salary inequities remain when controlling for age, specialty, hours worked, productivity, academic rank, and practice characteristics, with greater gaps for BIPOC and older women $[11,17,18]$. BIPOC women face structural inequities, particularly Black women, who have the greatest inequities in compensation, and generally have less familial wealth than White women, influenced by the Black-White wealth gap and other factors related to structural racism [5]. For example, Black parents are twice as likely to be unemployed and unmarried and are significantly less likely to own homes and more likely to single parent than White parents [5]. Between 2010 and 2013, annual median physician income adjusted for age, race, sex, hours worked, state of residence, and time period was $\$ 253,042$ for White men compared to $\$ 163,234$ for White women and $\$ 188,230$ for Black men compared to $\$ 152,784$ for Black women [18]. The interaction of characteristics such as sexual orientation, race/ethnicity, and gender identity have a compounding effect on salary inequities, yet are frequently omitted from salary discrepancy statistics [17], limiting our

Table 2 US MD clinical and psychiatry faculty salary mean compensation by rank and gender, 2013 vs. 2018*

\begin{tabular}{|c|c|c|c|c|c|c|c|c|c|c|}
\hline & \multicolumn{2}{|l|}{ Chair } & \multicolumn{2}{|l|}{ Professor } & \multicolumn{2}{|c|}{ Associate professor } & \multicolumn{2}{|c|}{ Assistant professor } & \multicolumn{2}{|c|}{ Clinical instructor } \\
\hline & 2013 & 2018 & 2013 & 2018 & 2013 & 2018 & 2013 & 2018 & 2013 & 2018 \\
\hline \multicolumn{11}{|c|}{ All Clinical Faculty } \\
\hline Male (\$) & 575,500 & 692,200 & 344,200 & 404,200 & 316,200 & 374,100 & 279,500 & 325,400 & 213,200 & 247,900 \\
\hline Female (\$) & 444,200 & 553,700 & 280,600 & 319,900 & 243,500 & 282,700 & 215,300 & 252,000 & 179,300 & 207,800 \\
\hline Difference $(\$)$ & 131,300 & 138,500 & 63,600 & 84,300 & 72,700 & 91,400 & 64,200 & 73,400 & 33,900 & 40,100 \\
\hline Difference $(\%)$ & $23 \%$ & $20 \%$ & $18 \%$ & $21 \%$ & $23 \%$ & $24 \%$ & $23 \%$ & $23 \%$ & $16 \%$ & $16 \%$ \\
\hline \multicolumn{11}{|l|}{ Psychiatry Faculty } \\
\hline Male $(\$)$ & 403,500 & 479,500 & 249,300 & 284,400 & 203,500 & 248,500 & 189,500 & 229,000 & 170,400 & 202,900 \\
\hline Female (\$) & 308,900 & 426,100 & 226,500 & 261,800 & 194,800 & 225,400 & 169,400 & 211,300 & 161,000 & 183,600 \\
\hline Difference (\$) & 94,600 & 53,400 & 22,800 & 22,600 & 8700 & 23,100 & 20,100 & 17,700 & 9400 & 19,300 \\
\hline Difference (\%) & $23 \%$ & $11 \%$ & $9 \%$ & $8 \%$ & $4 \%$ & $9 \%$ & $11 \%$ & $8 \%$ & $6 \%$ & $10 \%$ \\
\hline
\end{tabular}

Source: AAMC Faculty Salary Survey, MD or Equivalent Faculty by Gender, AAMC Data Services, April 2019

*Compensation included fixed/contractual salary, medical practice supplement, bonus pay, and uncontrolled outside earnings and excludes fringe benefits

*This analysis identifies high-level national trends, but should not be considered a complete analysis of faculty equity

*The national data set does not include numerous data points related to compensation, such as time in rank and productivity 
ability to cite empirical data. Still, less income during their working years will leave women with less retirement income, including social security and pension benefits [19].

\section{Overlapping Oppressions}

Within academic medicine, BIPOC women remain significantly more disadvantaged compared to White men and women and encounter compounded biases and barriers that thwart their advancement as they expend time and energy proving their authority and competence while simultaneously disproving harmful stereotypes [20]. This remains true in academic psychiatry. For example, a Black female psychiatrist, Dr. Baker, described experiencing racial slurs and other bigoted behavior, while White superiors silenced any exploration of racism [20]. While colleagues perpetuated harmful racebased generalizations about Black communities and their association with poverty and mental illness, Dr. Baker was forced to remain silent and expend time and energy addressing racist claims [20]. This example of navigating academic psychiatry while both Black and female further underscores the complexities of survival and career success for women with multiple minoritized statuses.

Prior research has documented women's susceptibility to stereotype threat (i.e., the anxiety faced when one is evaluated by negative stereotypes [21]). In a study in academic medicine, women junior faculty reported more vulnerability to stereotype threat, sensitivity to rejection, identification with their gender, feelings of lower relative potential, and a lower sense of belonging than their male counterparts [21].

BIPOC and LGBTQIA+ women are susceptible to stereotype threat and face unique challenges such as managing their visibility in organizational contexts in which they inhabit multiple minoritized statuses. Minoritized women risk becoming more visible because of their racial, sexual orientation, and gender differences rather than for their competence, character, or achievements [22]. Conversely, women trying to blend into the dominant narrative risk are becoming chronically invisible, silencing their achievements and contributions [22]. In addition, BIPOC and LGBTQIA+ women must navigate negative climate and culture characterized by racialized and sexist stereotypes at work, balancing frequent misperceptions of being perceived as aggressive or angry or not assertive enough, further silencing them [23]. This phenomenon is described as a double bind or jeopardy [23], which is even more compounded by feeling obligated or pressured to represent the minority perspective [24]. All women may experience sexism, bullying, and institutional roadblocks, but BIPOC, especially URM, and LGBTQIA+ women, are often more isolated and under-respected because of discrimination, tokenism, and the lack of diversity [13, 23-26].

\section{Harassment and Discrimination}

Women academic physicians of all identities are known to experience discrimination, gender-based harassment, unwanted sexual attention, and sexual coercion at an alarming frequency, even more so for BIPOC and LGBTQIA+ women $[27,28]$. A study of 5782 physician mothers found that $2 / 3$ reported gender-based discrimination and $1 / 3$ reported maternal discrimination, defined as discrimination due to pregnancy, maternity leave, or breastfeeding [29]. Salient examples of such discrimination, exacerbated for BIPOC women, include lower likelihood of being hired, promoted, or offered a leadership position [25, 30]. LGBTQIA+ women must face stigmatization, marginalization, and residency and job placement discrimination [26]. In prior studies, more than $50 \%$ of women faculty and staff in academic institutions reported harassment, commonly gender-based, perpetuated by faculty (superiors and peers) and patients, among others [27, 28]. Despite all women being susceptible to multiple forms of harassment, the experience between cisgender White women, BIPOC, and LGBGTQIA+ women are distinct [28].

BIPOC and LBGTQIA+ women are known to experience elevated rates of harmful overt and covert forms of overlapping oppression, harassment, and discrimination. These interdependent and compounded forms of oppression are deeply embedded in administrative and educational policies and practices, hindering women's progress and acting as mechanisms of social control [27, 28, 31]. LBGTQIA+ women face unique challenges as part of heteronormative cultures that are negative and invalidating. For example, LGBTQIA+ women physicians report being denied referrals and experiencing social ostracization while also experiencing delayed promotion, loss of practice or income, and negative stereotyping [26]. These inequities often include various forms of harassment, derogatory comments, and humiliation.

Additionally, one study found that BIPOC women in the sciences experienced more harassment (combining racial and sexual harassment) than White women, White men, and BIPOC men [28, 32]. Specifically, BIPOC women experienced more verbal racial harassment than other groups and equal rates of verbal sexual harassment compared to White women [28, 32] Further, LGBTQIA+ women experience higher rates of sexual harassment than heterosexual and cisgender women [32].

Research has documented that BIPOC and LGBTQIA+ women are less likely to report harassment than White women, due to fear of negative outcomes, retaliation, and beliefs that perpetrators will not be reprimanded [13, 27-29, 31]. Finally, women are not homogenous with mutually understood experiences. The differences in harassment and discrimination experienced by minoritized women remain unrecognized by White women, delegitimizing their experiences and perpetuating marginalization $[13,25]$. 


\section{Microaggressions and Bias}

Microaggressions can be described as "brief and commonplace daily verbal, behavioral and environmental indignities, whether intentional or unintentional, that communicate hostile, derogatory, or negative racial slights and insults to the target person or group" [33]. Microaggressions are not limited to race and are influenced by other minoritized statuses, such as ethnicity, gender, and sexual orientation. Microassaults, microinsults, and microinvalidations are subtle forms of oppression often more insidious and difficult to identify, further creating an impossible bind for BIPOC and LGBTQIA+ women who experience any variation of gendered, anti-gay, or racialized oppression [33]. Gender-based microaggressions predominately impact women, especially BIPOC and LGBTQIA+ women, in academia through three social mechanisms: gender blindness, gender-stereotypical assumptions, and sexual objectification [31].

Explicit and implicit bias (also known as unconscious bias) against women, including those with minoritized statuses, in medicine are well-documented and adverse factors in all career stages $[13,23,25,30]$. BIPOC women are recipients of automatic assumptions about their abilities and ambition; their work is often undervalued [13, 34]. Professional credibility and authority may be challenged regardless of their competence or ranking $[13,31]$. BIPOC women, especially Black women, commonly feel unsupported in medical school, while domestic born BIPOC women may hold fears based on perceived biases or xenophobic attitudes in clinical settings [23]. Asian-American women may fear being perceived as too young to hold the proper credentials [23]. These concerns translate into behavior and appearance changes, e.g., Muslim women stop wearing hijabs for fear of explicit and implicit bias related to Islamophobia [23]. Thus, implicit and explicit biases operate as a discriminatory form of social control. Additionally, such bias in psychiatry can be particularly troublesome as it likely influences students and trainees' assessment of presenting issues among ethnic and racial minoritized patients [35]. Taken together these findings may be correlated to the elevated rates of stress felt by BIPOC and LGBTQIA+ women [34].

\section{Minority Tax}

BIPOC faculty experience a phenomenon referred to as the minority tax which is defined as additional responsibilities and burdens placed on minoritized persons. They feel beholden to address racism, diversity, mentorship, clinical responsibilities for underserved populations, isolation, and promotion inequities [36]. Minority tax disrupts scholarly productivity by diverting a minoritized faculty's time to addressing systemic and structural problems, which are beyond their control [36]. Minority tax burdens are frequently experienced by those who are often isolated and may be one of few faculty members with a minoritized ethnic or racial identity [36]. Examples of minority tax include the following: designation as representative spokespersons for their racial group, assignment to offsite clinical work locations to serve health needs of marginalized communities, mentorship of a diverse cadre of students with inadequate mentorship, and assignment to institutional diversity efforts, often without compensation [36]. Minority tax is broad and all-encompassing and is harmful because it is inextricably tied to one's minoritized status, and often unfairly requires unrecognized and uncompensated labor [36].

\section{Impostor Syndrome}

Impostor syndrome is a psychological phenomenon, first described in high achieving women [37] that refers to a pattern of behavior wherein people doubt their abilities and persistently fear being exposed as a fraud, despite evidence of their success $[37,38]$. It is encountered in high stakes professions, including medicine and academic psychiatry. Some studies suggest higher severity in women faculty, especially BIPOC, due to added pressures to perform against racial and gender stereotypes and discrimination $[13,39]$. While men tend to own success as an inherent quality, such as being smart or creative, women may discount their success by stating that they worked hard or were lucky [37, 39].

A 2016 report showed that out of 138 medical students, $50 \%$ of female and $25 \%$ of male students were affected by impostor syndrome, which was less prevalent among White and Asian medical students than other race/ethnicities (30\% vs. 73\%) [40]. A systematic review of imposter syndrome among college students underscored a high prevalence among Black, Hispanic, and Asian students [39]. BIPOC, especially URM, students may be predisposed to imposter syndrome due to increased stress from sociodemographic, familial, and environmental factors, including racial discrimination and enduring negative stereotypes $[38,39]$. Impostor syndrome is correlated with anxiety, depression, burnout, physical exhaustion, and avoidance of tasks with high prominence [38, 39]. It has been associated with perfectionism, is a strong predictor of psychological distress, and may not abate with more years of training or experience $[38,39]$.

\section{Lack of Mentorship/Sponsorship}

Mentorship and sponsorship are integral components of one's career advancement [41], yet male physicians are more likely to receive mentorship or sponsorship than females or BIPOC [42-44]. Women are also less likely than men to have mentors/sponsors in power positions, which reduce their access to career-promoting opportunities. Further, the majority of institutions lack specific programs targeting women and BIPOC [42-44]. URM faculty especially rely on mentorship 
for success, yet the lack of representation of senior URM faculty translates into mentorship remaining a barrier to recruitment, retention, and advancement [8]. Further, sponsorship is important for the advancement of URM women into leadership roles, as they may be more subject to implicit bias and discrimination than their White counterparts [20, 22, 23].

\section{Work Life Integration}

Work life integration with domestic and caregiving responsibilities creates barriers and challenges for women's advancement. One report found that childbearing leave (i.e., for birth mothers) and paid family leave (i.e., for post-birth or non-birth parents) policies are inconsistent across top medical universities [44]. Although all twelve universities studied provided childbearing leave, only three provided full salary support for more than 8 weeks and only eight allowed extensions [44]. The mean paid family leave duration was approximately 18 weeks; only four universities provided more than 8 weeks of full salary support [44]. Parental leave during residency creates further barriers due to minimum training requirements and difficulty obtaining fellowship and academic positions "off cycle" [45]. Additional barriers arise upon returning to work as child care and lactation support can be nearly impossible to find even though the USA guarantees breastfeeding breaks $[46,47]$.

These challenges are more complex for URM women, who may be first-generation college or professional school graduates, may be supporting their families of origin including their own, and often have less familial wealth by which to establish financial stability, especially Black women, as described previously [5]. Further, only half of Black college graduates in 2014 were married compared to $59 \%$ of Hispanic college graduates, $68 \%$ of White college graduates, and $74 \%$ of Asian college graduates, and those who marry have more marital instability than other groups, known as the Black Marriage Gap [5]. These findings underscore how inconsistent and rigid policies may pose additional challenges to the retention of women and primary caregivers, especially URM, in the workforce and further explain the gender wage gap [44].

\section{Promising Solutions for the Advancement of Women}

\section{Dismantle Harassment and Implicit Bias}

To address the pervasiveness of harassment across medical fields, the National Academies Press (2018) has recommended the following interventions: (1) diffusion of hierarchical and dependent relationships between trainees and faculty; (2) provision of support for targets of harassment; (3) improvement of transparency and accountability; and (4) empowerment of the entire academic community with responsibility to reduce and prevent sexual harassment [28].

Institutional leadership must promote change to dismantle harassment and bias. We cannot wait until sufficient women and BIPOC are promoted to institutional leadership to address these urgent concerns. In academic medicine, sexual and racial harassment identification and response training along with a systemwide changing of cultures and climates that target harassment and reprimand these behaviors may create safer environments for all women and thus improve diversity among academic physicians [27]. Training and awareness about implicit and explicit bias manifestations and interventions; creation of diverse, inclusive, and respectful environments; and integration of these entities into policies and procedures may be especially helpful [27].

Purposefully developing safer and more equitable environments for all women, and especially BIPOC and LGBTQIA+ women, may include the following: enacting open door and zero tolerance policies that validate women's experiences and target retaliation or other negative outcomes; developing equity, diversity, and inclusion committees to monitor and hold institutions accountable for systemic discriminatory practices; and creating supportive cultures where colleagues feel emboldened to speak out against and report harassment. [27, 28]

\section{Eliminate Gender Pay Gap}

Academic institutions should utilize pay scale and promotion criteria transparency from the initial appointment, periodically review compensation and promotion rates by gender, and race/ethnicity evaluate advancement protocols for potential discrimination $[16,41]$. Equity review should include all aspects of compensation; should be based on competencies, expertise, and not on personal characteristics; and should not include part-time penalties [19, 41]. Medical schools that have conducted comprehensive salary equity reviews and have tackled salary disparity utilizing multiple points of data to set benchmarks, conducting reviews of outliers, and presenting their data broadly to stakeholders at multiple levels report improved perceptions of transparency and salary equity within their institutions [17]. Salary equity interventions are especially important for URM women, and most significantly Black women, who have the greatest gender salary gap, experience more structural racism, and have less personal and familial wealth as previously noted [5].

\section{Address Impostor Syndrome}

Prior research has documented several strategies to address impostor syndrome at individual and institutional levels. Individual strategies include the following: (1) developing self-awareness regarding impostor syndrome; (2) creating a 
personal career success inventory; (3) writing down steps taken to earn achievements; (4) celebrating accomplishments; (5) maintaining a record of positive feedback; (6) practicing thought stopping and challenging cognitive distortions; and (7) seeking mentors and sponsors [38]. Institutional strategies may include educational workshops for medical students and faculty aimed at early recognition of the impostor phenomenon and targeted mentorship programs, especially for URM [38]. Tips for nurturing a growth mindset include providing multifaceted feedback, viewing struggle as opportunity, praising an attempt at a new approach and well-directed effort instead of intelligence alone, and encouraging self-reflection with goal setting [38].

\section{Enhance Mentorship and Sponsorship Opportunities}

Academic institutions should develop formal mentorship and sponsorship programs designed to address underrepresentation for women, prioritizing URM women [42]. Programs may utilize multiple mentoring formats [48] and should set goals for quality of mentorship/sponsorship experiences [49-51], number/quality of participants' scholarly activities [52], and success of advancement and retention. Progress toward goals should be regularly evaluated, and programs should be adequately staffed and funded $[42,53]$.

Departmental leaders (e.g., chairs, chiefs, team leaders) as well as faculty mentors and sponsors should undergo diversity, equity, and inclusion training, including implicit and explicit bias training. Departments should prioritize, recognize, and financially support faculty for mentorship and sponsorship activities, especially to support URM women [53]. Leaders should create organizational cultures that openly elicit feedback and address concerns related to minority tax when identifying minoritized faculty as mentors/sponsors. We stress the importance of incentivizing and rewarding faculty participation by protecting their time, ensuring they have adequate training and resources, and acknowledging exceptional performance through merit awards, fellowships, or bonuses. Leaders are also well placed to sponsor faculty and promote sponsorship systemically among URM women, given their connections and elevated status. URM women faculty should also be supported in accessing extramural mentorship/sponsorship opportunities through national professional societies and organizations.

Peer mentoring programs should also be established within departments of psychiatry to further address mentoring barriers, to enhance faculty collaboration, to increase productivity, and to change institutional policies that perpetuate discrimination, bias, and inequity $[30,52,54]$. Programs that additionally provide ongoing social and professional support, decrease isolation and burnout, develop a sense of community, and build the core of the group's professional network are especially important for URM women to build cohesion with other women faculty, as they may be the only one or one of few with their minoritized status or statuses within their departments $[30,52,54]$.

\section{Create Responsive Caregiving Programs}

Women's ability to pursue careers and leadership while balancing competing demands of childcare and family needs may be judged differently based on institutional cultures and may be particularly challenging for URM women faculty, who are already expending extra energy to overcome bias, harassment, and discrimination as described previously. To overcome these challenges, paid parental leave, part-time options, flexible schedules, job-sharing, work at home options, onsite lactation support, and child care programs are critical to support parents from medical students to residents to faculty $[41,44,45]$.

The benefits of breastfeeding and maternal/paternal leave are well known and far outweigh the cost of lost productivity and job satisfaction [47]. Mandatory paid childrearing and parental leave of 12 weeks would significantly ease the burden of physician parents with optional paid or unpaid leave up to 6 months to support healthy child development [41, 45, 52]. Paid leave is critically important for URM, who are less likely to have financial reserves available to take unpaid leave [5]. Further, for residents, leave makeup time requirements should be abandoned in favor of competency assessments, and the use of sick leave should not be required [47]. Breastfeeding policies should be flexible and provide greater sense of caregiver agency [45, 47, 55]. Onsite childcare availability should be standardized or at least subsidized as an incentive for recruitment and retention, especially for URM faculty $[5,47]$. On the other end of the lifespan, paid sick and family leave policies play a critical role to relieve the growing burden and costs of family caregiving of older adults [56]. Such policies, along with flexible schedules, could go a long way toward increasing engagement, satisfaction, and retention and are important to advance the careers of women, especially URM, faculty [41, 47, 55].

\section{Final Thoughts}

In conclusion, while addressing equity concerns is important for all women, a special focus on the needs of BIPOC, especially URM, and LGBTQIA+ women is important due to their intersecting identities and underrepresentation as trainees, faculty, and senior positions in academic psychiatry and requires a multipronged approach. It is imperative that academic institutions account for the challenges encountered by women in balancing their domestic and professional roles. Additionally, recognition of the distinct, multifactorial disparities that exist 
for URM and LGBTQIA+ women is critically important. These women are further marginalized when discourse focuses on gender without accounting for the structural inequities caused by racism and other forms of discrimination and bias based on marginalized statuses.

Notably, emergent research and institutional policies and programming would benefit from more empirical data and in-depth analysis on the compounded oppressions faced by URM, especially Black, women in academic psychiatry. In the wake of the Black Lives Matter movement, academic medicine has an obligation to move beyond performative statements and symbolic efforts into explicit action [57]. Though sparse, emergent research has suggested that academic medicine may incorporate explicitly antiracist curricula, committees, and policies into institutional changes by actively listening to those impacted by racial inequities [57]. Educational institutions, including academic psychiatry departments, should then implement effective solutions that dismantle oppressive structures.

Solutions should include the development of carefully designed recruitment tactics, mentorship programs and sponsorship opportunities, responsive caregiving programs, and creative pathways to address implicit and explicit bias, sexual harassment, and salary inequities. Recruitment strategies may focus on improving outreach to diverse groups of women, enhancing educational curricula to be more inclusive and culturally competent, and expanding opportunities that are relevant to the clinical and research interests of these groups [4]. Mentorship programs targeting URM women faculty are critically needed [58] and are an important mechanism to address gender and racial bias. Such solutions require intentional acts to increase the availability of child care, flexible work schedules, and part-time work; provide education to search committee members; and address systemic oppression. Further, it is imperative that these policies, protocols, and programs are both known and accessible among all women, which may further dismantle barriers to achieving equity. Educational institutions must take into consideration the recruitment, promotion, and retention of diverse female faculty and specifically account for how racism, sexism, and other forms of discrimination hinder progress.

Acknowledgments Bridging the Gender and Diversity Gap: Mentorship Models for Addressing Under-representation for Women Psychiatrists, Annual Meeting of the American Academy of Child, and Adolescent Psychiatry, Chicago, Illinois, 2019.

\section{Compliance with Ethical Standards}

Disclosure On behalf of all of the authors, the corresponding author states that there is no conflict of interest.

Ethical Approval None.

\section{References}

1. Heisers S. The majority of U.S. medical students are women, new data show. AAMC. 2019. https://www.aamc.org/news-insights/ press-releases/majority-us-medical-students-are-women-new-datashow. Accessed 20 May 2020.

2. Schor NF. The decanal divide: women in decanal roles at U.S. medical schools. Acad Med. 2018;93:237-40.

3. Faculty roster: U.S. medical school faculty. AAMC. 2019. https://www.aamc.org/data-reports/faculty-institutions/ interactive-data/2019-us-medical-school-faculty. Accessed 14 October, 2020.

4. Pierre JM, Mahr F, Carter A, Madaan V. Underrepresented in medicine recruitment: rationale, challenges, and strategies for increasing diversity in psychiatry residency programs. Acad Psychiatry. 2017;41(2):226-32.

5. On views of race and equality, blacks and Whites are worlds apart. Pew Research Center, Social and Demographic Trends. 2016. https://www.pewsocialtrends.org/2016/06/27/1 demographic-trends-and-economic-well-being. Accessed October 10, 2020.

6. Underrepresented in Medicine Definition. AAMC. 2004. https:// www.aamc.org/what-we-do/mission-areas/diversity-inclusion/ underrepresented-in-medicine. Accessed 25 February 2020.

7. Jagsi R, Griffith KA, Stewart A, Sambuco D, DeCastro R, Ubel PA. Gender differences in the salaries of physician researchers. JAMA. 2012;307(22):2410-7.

8. Chaudhary AMD, Naveed S, Siddiqi J, Mahmood A, Khosa F. US psychiatry faculty: academic rank, gender and racial profile. Academic psychiatry. Acad Psychiatry. 2020;44(3):260-6.

9. Lewin MR. Pregnancy, parenthood, and family leave during residency. Ann Emerg Med. 2003;41(4):568-73.

10. Jagsi R, DeCastro R, Griffith KA, Rangarajan S, Churchill C, Stewart A, et al. Similarities and differences in the career trajectories of male and female career development award recipients. Acad Med. 2011;86(11):1415-21.

11. Raj A, Kumra T, Darmstadt L, Freund KM. Achieving gender and social equality: more than gender parity is needed. Acad Med. 2019;94(11):1658-64.

12. Jagsi R, Means $\mathrm{O}$, Lautenburger D, Jones RD, Griffith KA, Flotte TR, et al. Women's representation among members and leaders of national medical specialty societies. Acad Med. 2020;95(7):1043-9.

13. Turner CSV. Women of color in academe: living with marginality. J High Educ. 2002;73(1):74-93.

14. Mensah M, Beeler W, Rotenstein L, Jagsi R, Spetz J, Linos E, et al. Sex differences in salaries of department chairs at public medical schools. JAMA Intern Med. 2020;180(5):789-92.

15. Faculty salary survey, total compensation in thousands of dollars, all medical schools, MD or equivalent faculty by gender. AAMC. 2019. https://www.aamc.org/system/files/2019-09/ aamcfacultysalarydata-md.pdf. Accessed 11 October 2020.

16. Bates C, Gordon L, Travis E, Chatterjee A, Chaudron L, Fivush B, et al. Striving for gender equity in academic medicine careers: a call to action. Acad Med. 2016;91(8):1050-2.

17. Dandar VM, Lautenberger DM, Garrison GE. Promising practices for understanding and addressing salary equity at U.S. medical schools. AAMC. 2019. https://www.aamc.org/data-reports/ faculty-institutions/report/promising-practices-understanding-andaddressing-salary-equity-us-medical-schools. Accessed September 28, 2020.

18. Ly DP, Seabury SA, Jena AB. Differences in incomes of physicians in the United States by race and sex: observational study. BMJ. 2016;353:i2923. 
19. Butkus R, Serchen J, Moyer DV, Bornstein SS, Hingle ST, Health and Public Policy Committee of the American College of Physicians. Achieving gender equity in physician compensation and career advancement: a position paper of the American College of Physicians. Ann Intern Med. 2018;168(10):721-3.

20. Baker FM. The black woman academic psychiatrist. Acad Psychiatry. 1993;17(4):194-201.

21. Fassiotto M, Hammel EA, Ku M, Carroll S, Grewal D, Lavori P, et al. Women in academic medicine: measuring stereotype threat among junior faculty. J Women's Health. 2016;25(3):292-8.

22. Blake-Beard SD, Roberts LM. Releasing the double bind of visibility for minorities in the workplace. Center for Gender in Organizations, Simmons School of Management; 2004.

23. Yang J. For women of color in medicine, the challenges extend beyond education. In Zora, 2019. https://zora.medium.com/forwomen-of-color-in-medicine-the-challenges-extend-beyondeducation-4df4e4b78b58. Accessed 20 May 2020.

24. Roberts LW, Maldonado Y, Coverdale JH, Balon R, Louie AK, Beresin EV. The critical need to diversify the clinical and academic workforce. Acad Psychiatry. 2014;38(4):394-7.

25. Albert M. \#Me who anatomy of scholastic, leadership, and social isolation of underrepresented minority women in academic medicine. Circulation. 2018;138(5):451-4.

26. Mansh M, Garcia G, Lunn MR. From patients to providers: changing the culture in medicine to sexual and gender minorities. Acad Med. 2015;90(5):574-80.

27. Golden SH. The perils of intersectionality: racial and sexual harassment in medicine. J Clin Invest. 2019;130(9):3465-7.

28. National Academies of Sciences, Engineering, and Medicine. Sexual harassment of women: climate, culture, consequences in academic sciences, engineering, medicine. Washington, DC: National Academies Press; 2018. https://doi.org/10. $17226 / 24994$.

29. Adesoye T, Mangurian C, Choo EK, Girgis C, Sabry-Alnagger H, Linos E, et al. Perceived discrimination experienced by physician mothers and desired workplace changes: a cross-sectional survey. JAMA Intern Med. 2017;177(7):1033-6.

30. Mangurian C, Linos E, Sarkar U, Rodriquez C, Jagsi, R. What's holding women in medicine back from leadership. Harvard Business Review. 2018. https://hbr.org/2018/06/whats-holdingwomen-in-medicine-back-from-leadership. Accessed 20 February 2020.

31. Carroll, D. A faculty woman of color and micro-invalidations at a White research institution: a case of intersectionality and institutional betrayal. Administrative Issues Journal https://dc.swosu.edu/aij/ vol7/iss 1/4/. Accessed 1 December 2020.

32. Clancy KBH, Lee KMN, Rodgers EM, Richey C. Double jeopardy in astronomy and planetary science: women of color face greater risks of gendered and racial harassment. Journal of Geophysical Research: Planets. 2017;122:1610-23. https://doi.org/10.1002/ 2017JE005256.

33. Sue DW, Capodilupo CM, Torino GC, Bucceri JM, Holder AMB, Nadal K, et al. Racial microaggressions in everyday life: implications for clinical practice. Am Psychol. 2007;62(4): 271-86.

34. Council NR, Affairs $\mathrm{P}$ and $\mathrm{G}$, Medicine $\mathrm{C}$ on $\mathrm{W}$ in $\mathrm{S}$ Engineering, and, Academia C on AIT for MW in. Seeking solutions: maximizing American talent by advancing women of color in academia: summary of a conference. Washington DC: National Academies Press; 2013.

35. Fadus MC, Odunsi OT, Squeglia LM. Race, ethnicity, and culture in the medical record: implicit bias or patient advocacy? Acad Psychiatry. 2019;43(5):532-6.

36. Rodríguez JE, Campbell KM, Pololi LH. Addressing disparities in academic medicine: what of the minority tax? BMC Med Educ. 2015;15(6). https://doi.org/10.1186/s12909-015-0290-9.
37. Clance PR, Imes S. The impostor phenomenon in high achieving women: dynamics and therapeutic interventions.Psychotherapy: theory. Research \&Practice. 1978;15:241-7.

38. Seritan AL, Mehta MM. Thorny laurels: the impostor phenomenon in academic psychiatry. Acad Psychiatry. 2016;40(3): 418-21.

39. Bravata DM, Watts SA, Keefer AL, Madhusudhan DK, Taylor KT, Clark DM, et al. Prevalence, predictors, and treatment of impostor syndrome: a systematic review. J Gen Intern Med. 2020;35(4): 1252-75.

40. Villwock JA, Sobin LB, Koester LA, Harris TM. Impostor syndrome and burnout among American medical students: a pilot study. Int J Med Educ. 2016;7:364-9.

41. Principles for advancing gender equity in medicine. American Medical Association. 2019. https://policysearch.ama-assn.org/ policyfinder/detail/Principles\%20for\%20Advancing $\% 20$ Gender $\%$ 20Equity\%20in\%20Medicine?uri=\%2FAMADoc\%2FHOD.xmlH-65.961.xml. Accessed 24 May 2020.

42. Carr PL, Gunn C, Raj A, Kaplan S, Freund KM. Recruitment, promotion, and retention of women in academic medicine: how institutions are addressing gender disparities. Womens Health Issues. 2017;27(3):374-81.

43. Farkas AH, Bonifacino E, Turner R, Tilstra SA, Corbelli JA. Mentorship for women in academic medicine: a systematic review. J Gen Intern Med 2019; 34(7):1322-9

44. Riano NS, Linos E, Accurso EC, Sung D, Linos E, Simard JF, et al. Paid family and childbearing leave policies at top US medical schools. JAMA. 2018;319(6):611-4.

45. Weinstein DF, Mangurian C, Jagsi R. Parenting during graduate medical training: practical policy solutions to promote change. New Eng J Med. 2019;381(11):995-7.

46. Earle A, Mokomane Z, Heymann J. International perspectives on work-family policies: lessons from the world's most competitive economies. Futur Child. 2011;21(2):191-210.

47. Itum DS, Oltmann SC, Choti MA, Piper HG. Access to paid parental leave for academic surgeons. J Surg Res. 2019;233:144-8.

48. Geraci SA, Thigpen SC. A review of mentoring in academic medicine. Am J Med Sci. 2017;353(2):151-7.

49. Palepu A, Friedman RH, Barnett RC, Carr PL, Ash AS, Szalacha L, et al. Junior faculty members' mentoring relationships and their professional development in U.S. medical schools. Acad Med. 1998;73(3):318-23.

50. Straus SE, Chatur F, Taylor M. Issues in the mentor-mentee relationship in academic medicine: a qualitative study. Acad Med. 2009;84(1):135-9.

51. Straus SE, Johnson MO, Marquez C, Feldman MD. Characteristics of successful and failed mentoring relationships: a qualitative study across two academic health centers. Acad Med. 2013;88(1):82-9.

52. Petrovic-Dovat L, Forgey Borlik M, Wadell P, Bath EP. Bridging the gender and diversity gap: mentorship models for addressing under-representation for women psychiatrists. 2019; Proceedings of the annual meeting of the American Academy of Child and Adolescent Psychiatry, Chicago, IL.

53. Sambunjak D, Straus SE, Marusic A. Mentoring in academic medicine: a systematic review. JAMA. 2006;296(9):1103-15.

54. Seritan AL, Bhangoo R, Garma S, Dube J, Park JH, Hales R. Society for women in academic psychiatry: a peer mentoring approach. Acad Psychiatry. 2007;31(5):363-6.

55. Morain S, Schoen L, Marty M, Bimla SE. Parental leave, lactation, and childcare policies at top US schools of public health. Am J Public Health. 2019;109(5):722-8.

56. Chen ML. The growing costs and burden of family caregiving of older adults: a review of paid sick leave and family leave policies. Gerontologist. 2016;56(3):391-6. 
57. Yancy CW. Academic medicine and black lives matter: time for deep listening. JAMA. 2020;324(5):435-6.

58. Kosoko-Lasaki O, Sonnino RE, Voytko ML. Mentoring for women and underrepresented minority faculty and students: experience at two institutions of higher education. J Natl Med Assoc. 2006;98(9): $1449-59$.
Publisher's Note Springer Nature remains neutral with regard to jurisdictional claims in published maps and institutional affiliations. 